\title{
Specialized clinical cytology may improve the results of EUS (endoscopic ultrasound)-guided fine-needle aspiration (FNA) from pancreatic tumors
}

Authors

Institutions
Arne R. Schneider ${ }^{1}$, Andreas Nerlich ${ }^{2}$, Theodoros Topalidis ${ }^{3}$, Wolfgang Schepp

Institutions are listed at the end of article. submitted 31. August 2014 accepted after revision 22. September 2014

\section{Bibliography}

Dol http://dx.doi.org/

10.1055/s-0034-1390886

Published online: 5.12.2014

Endoscopy International Open 2015; 03: E134-E137

(c) Georg Thieme Verlag KG

Stuttgart · New York

E-ISSN 2196-9736

\section{Corresponding author}

Arne Schneider, MD

Department of

Gastroenterology, Hepatology, and Gastroenterological Oncology

Bogenhausen Academic

Teaching Hospital

Englschalkinger Str. 77

D-81925 Munich

Germany

Fax: +4989/9270-2486

arne.schneider@|rz.tum.de
Background and study aims: A variety of factors (needle type, needle passes, tumor location, cytological assessment, etc.) may influence the diagnostic accuracy of endoscopic ultrasound-guided fine-needle aspiration cytology (EUS-FNAC) from pancreatic tumors. Whereas most published studies report a diagnostic accuracy of $>80 \%$ for EUS-FNAC, the results in routine settings are often considerably lower. This retrospective study aimed to define the effect of switching microscopic assessment from a standard pathology department to a highly specialized institute of cytology.

Patients and methods: A total of 63 patients underwent EUS-FNAC of solid or semisolid pancreatic masses. Specimens of the first consecutive 20 cases (Phase 1 ) were assessed by the local department of pathology. Then in Phase 2, involving another 43 subsequent cases, a specialized cytol-

\section{Introduction}

Endoscopic ultrasound-guided fine-needle aspiration (EUS-FNA) is an established means for the diagnostic workup of pancreatic masses $[1,2]$. Since its introduction into clinical medicine, the technique has been refined and different needle types have been compared with regard to their diagnostic gain. Numerous studies report accuracy and sensitivity of EUS-FNA cytology to reach up to $95 \%$. However, there is a large variation in these statistical results, ranging from 68.1 to $95 \%$ [3-10]. The reasons for this discrepancy remain ambiguous, although the reality in many endoscopy departments is that many physicians are dissatisfied with the accuracy of EUS-FNAC in their clinical routines. The technique of tissue sampling via EUS-guided FNA is well defined and largely standardized. The question of preference for small (25-gauge), intermediate (22-gauge), or large-size (19-gauge) needles - or whether to use core biopsy needles, with a chance of obtaining a ogy laboratory examined all aspirates. All EUSFNACs were performed in the same manner, using a 22-gauge needle. After cytological evaluation, all patients either underwent surgery or were followed up for at least 6 months.

Results: Of the tumors, 56 were solid and 7 semisolid; the mean size was $30 \mathrm{~mm}$. Sensitivity (sens.), specificity (spec.), positive predictive value (PPV), and negative predictive value (NPV) of EUS-FNAC were $38.5 \%$ (95\%CI [confidence interval] $13.9-68.4 \%), 100 \% \quad(59.0-100 \%), \quad 100 \%$ (47.8-100\%), and $46.7 \%(21.3-73.4 \%)$ during Phase 1 versus $91.4 \%$ (95\%CI 76.9-98.2\%), 100\% (63.1-100\%), $100 \%$ (89.1-100\%), and $72.7 \%$ (39.0-94.0\%) during Phase 2.

Conclusion: These results emphasize the considerable impact of a dedicated cytological evaluation on the results of EUS-FNAC.

histological diagnosis - seems to remain a matter of ongoing debate [3,11-15].

Once successful aspiration has been achieved, further processing of the specimen by endoscopists and assessment by cytopathologists seem highly variable: Some physicians prefer formalin fixation to enable histopathological embedding, whereas the majority prepare a varying number of slides that undergo either air drying or chemical fixation. Additionally, cytopathologists employ a wide range of staining methods, depending on individual preferences.

During past years, we have modified several of the abovementioned aspects to improve the sensitivity of EUS-FNAC in our department. However, none of these merely technical modifications yielded a detectable increase in sensitivity.

We therefore agreed on switching the microscopic assessment of the slides from our in-house department of pathology to a dedicated laboratory of clinical cytology. The present study compares the results of EUS-FNAC of solid or semiso- 
lid pancreatic masses performed at our department before and after this modification.

\section{Patients and methods}

$\nabla$

The study population comprised all patients undergoing EUSFNA at our department for diagnostic workup of solid or semisolid (cystic with solid fractions) pancreatic masses between January 2009 and September 2012. Patients were identified retrospectively by using the electronic medical information database of the hospital. Most patients underwent EUS-FNA for cytological confirmation of suspected unresectable adenocarcinoma of the pancreas. In contrast, patients with highly suspicious but potentially resectable tumors were sent directly for surgery without pursuing a preoperative microscopic diagnosis. Individual courses of disease were followed for a minimum of 6 months, particularly to rule out false-negative results of cytology where pancreatic cancer might evolve despite nonmalignant cytology. In the latter case, lesions were considered malignant either if a carcinoma was histologically confirmed after resection, if the size of the lesion increased during follow-up, or if its size decreased during chemotherapy.

EUS-FNA was performed using a curvilinear array echoendoscope (Pentax EG-3870UTK, Pentax Medical, Hamburg, Germany) attached to a Hitachi EUB 8500 ultrasound processor (Hitachi Medical Systems, Wiesbaden, Germany). The procedure was performed by experienced endoscopists ( $>30$ previous procedures) with patients in the left lateral position under sedation with intravenous propofol. For tumors of the pancreatic head, puncture was done from the duodenal position in the majority of patients. The remaining patients were visualized and biopsied using a transgastric approach. All EUS-FNA were performed with a 22gauge needle (Sonotip II, Medi-Globe, Achenmühle, Germany). After visualization of the tumor in a stable position of the endoscope, the needle was advanced into the tumor under continuous EUS guidance, then the stylet was entirely removed and suction applied. A minimum of six needle passes to and fro were performed inside of the lesion during two to four separate punctures, depending on the macroscopic appearance of the specimen with respect to cellularity and bloody additives. Further punctures were abandoned when gross inspection augured tissue without relevant bloody admixture. The aspirate was then recovered by reintroduction of the stylet and subsequent sparging of the needle with air from a 5-mL luer lock syringe. Then smears on glass slides were readily prepared from aspirated fluid with corpuscular fractions. Additionally, cellular clots or spools were gently rolled over glass slides to allow for a detachment of cells on the slide surface. The slides were finally air dried and sent for further cytopathological evaluation.

During study Phase 1 from January 2009 through October 2010, dried aspirates were sent for microscopic diagnosis to the hospital's institute of pathology. There, the slides were stained with Giemsa and analyzed for adequacy of the specimen, cellularity, blood cell additives, and final diagnosis. After this period (Phase 2, November 2010-September 2012), the slides were sent to a specialized institute of clinical cytology. In contrast, May-Grünwald-Giemsa was the standard staining method of the cytologist, whereas further microscopic assessment of cytological aspirates corresponded to the workflow outlined above. Sensitivity (sens.), specificity (spec.), positive predictive value (PPV), and negative predictive value (NPV) as well as corresponding $95 \%$ confidence interval (CI) were calculated with GraphPad Prism 6 (GraphPad Software Inc., La Jolla, CA, USA). The study was approved by the local ethics committee (Ethics Committee of the Faculty of Medicine, Technical University Munich; approval no. 588/13).

\section{Results \\ $\nabla$}

Of 64 consecutive patients who had undergone EUS-FNAC, aspirates of 63 patients (24 male [38\%], 39 female [62\%]; mean age 70 , range $28-85$ ) showed cytological features of pancreatic parenchyma and were included in the study. During Phase 1, 20 patients underwent EUS-FNAC; in Phase 2, patients undergoing the procedure numbered 43 . The tumor sites of the entire group were: 35 tumors (56\%) located in the head; 21 tumors (33\%) in the body; and 7 tumors (11\%) in the tail of the pancreas. When the study population was differentiated according to affiliation (Phase 1/Phase 2 of the study period), the distribution of the sites broke down to: $5 / 16(25 / 37 \%)$ in the head; $11 / 22(55 / 51 \%)$ in the body; and 4/5 (20/12\%) in the tail. Lesions were classified hypoechoic in 56 cases (89\%) and hypoechoic with cystic areas in 7 cases $(11 \%)$. The mean size of the tumors in the entire population was $30 \mathrm{~mm}$ (range $12-80 \mathrm{~mm}$ ), with $39 \mathrm{~mm}$ (range $12-80 \mathrm{~mm}$ ) in the Phase 1 group, and $30 \mathrm{~mm}$ (range $13-70 \mathrm{~mm}$ ) in the Phase 2 group ( $P=0.12$, Mann-Whitney test). During EUS-FNAC, 2 to 5 separate needle passes provided 4 to 27 slides with smears. Mean follow-up of all patients was 17.8 months (range 6.2-34.7 mo).

Surgical resection was done on 9 patients and 54 patients were solely followed up. On final diagnosis, 48 lesions were malignant (44 adenocarcinoma, 3 neuroendocrine tumors [NET], and 1 metastasis of breast carcinoma); and 15 lesions were of benign origin ( 2 focal post-inflammatory residues after acute pancreatitis, 2 focal inflammatory changes within chronic pancreatitis, 1 autoimmune pancreatitis, 2 intraductal papillary mucinous neoplasms [IPMN], 3 hemorrhagic pancreatic cysts, and 5 undefined).

Accordingly, sens., spec., PPV, and NPV of EUS-FNAC were $38.5 \%$ (95\%CI 13.9-68.4\%), 100\% (59.0-100\%), 100\% (47.8-100\%), and $46.7 \%(21.3-73.4 \%)$ during Phase 1 versus $91.4 \%$ (95\%CI $76.9-98.2 \%), 100 \%$ (63.1-100\%), 100\% (89.1-100\%), and $72.7 \%$ (39.0-94.0\%) during Phase 2 ( Table 1). Accuracy was 60 and $93 \%$, respectively.

\section{Discussion \\ $\nabla$}

Published data report on a high accuracy of EUS-FNAC for the diagnosis of pancreatic tumors. According to a variety of studies, mean sensitivity, specificity, and accuracy can be expected to be around 83,100 , and $88 \%$ - although these statistical parameters may vary from 54 to $95 \%, 71$ to $100 \%$, and 65 to $96 \%$, respectively [16-18].

However, clinical experience among physicians applying the technique in routine diagnostics is often considerably lower than these study results. Some endoscopists may even have abandoned EUS-guided fine-needle aspiration because, due to ambiguous cytology, they lack faith in the results. In this regard, several factors may have an effect on the diagnostic yield of EUSFNAC. 
Table 1 Summary of statistical results.

\begin{tabular}{|c|c|c|c|c|c|c|c|}
\hline \multirow[t]{3}{*}{ Phase $1(n=20)$} & \multicolumn{7}{|c|}{ Phase $2(n=43)$} \\
\hline & \multicolumn{2}{|c|}{ Real result } & \multirow[t]{2}{*}{$\Sigma$} & & \multicolumn{2}{|c|}{ Real result } & \multirow[t]{2}{*}{$\Sigma$} \\
\hline & Benign & Mal & & & Benign & Malignant & \\
\hline FNAC+ & 0 & 5 & 5 & FNAC+ & 0 & 32 & 32 \\
\hline FNAC- & 7 & 8 & 15 & FNAC- & 8 & 3 & 11 \\
\hline$\Sigma$ & 7 & 13 & 20 & $\Sigma$ & 8 & 35 & 43 \\
\hline Sens. & \multicolumn{3}{|c|}{$38.5 \%(13.9-68.4 \%)$} & Sens. & \multicolumn{2}{|c|}{$91.4 \%(76.9-98.2 \%)$} & \\
\hline Spec. & \multicolumn{3}{|c|}{$100 \%(59.0-100 \%)$} & Spec. & \multicolumn{2}{|c|}{$100 \%(63.1-100 \%)$} & \\
\hline PPV & \multicolumn{3}{|c|}{$100 \%(47.8-100 \%)$} & PPV & \multicolumn{2}{|c|}{$100 \%(89.1-100 \%)$} & \\
\hline NPV & \multicolumn{3}{|c|}{$46.7 \%(21.3-73.4 \%)$} & NPV & \multicolumn{2}{|c|}{$72.7 \%(39.0-94.0 \%)$} & \\
\hline
\end{tabular}

Abbreviations: FNAC, fine-needle aspiration cytology benign; FNAC+, fine-needle aspiration cytology malignant; NPV, negative predictive value; PPV, positive predictive value; Sens., sensitivity; Spec., specificity.

$95 \%$ confidence interval.

The endoscopic technique of EUS-FNA has been well defined and even outlined in clinical guidelines. A minimum of two to five needle passes per lesion, depending on a gross macroscopic assessment of the aspirate by the endoscopist, are considered mandatory for adequate microscopic diagnosis [18]. The question of whether to use smaller (25-gauge) or larger size (19-, 22-gauge) or even core biopsy needles is still a matter of debate $[3,11,12]$. However, conflicting results of these studies suggest a minor effect of this issue. Immediate microscopic assessment of aspirates by an on-site cytopathologist may further improve EUS-FNAC and reduce the need for multiple needle passes; however, this is rarely available in the routine setting [19-21]. If the initial EUSFNAC is not diagnostic, repeating the procedure may improve results $[19,20]$. Alternatively, percutaneous computed tomography (CT) or ultrasound-guided puncture can be performed, but implies a higher risk for peritoneal tumor seeding. There is no evidence that larger tumors facilitate FNAC assessment [22]. This may derive from the fact that the larger the lesion, the more often nondiagnostic aspirates from cystic or necrotic areas are encountered.

Regarding microscopic diagnosis, cytopathologists generally consider pancreatic adenocarcinoma a challenging task because scarce cellularity, inflammation, and desmoplastic changes may mimic both well-differentiated carcinoma in chronic pancreatitis and fibrosing pancreatitis in cancers $[17,23,24]$. Since standardized guidelines defining the cytological workup are lacking, preparation and further processing of slides remain highly individual. Whereas some cytopathologists prefer air-dried samples, others recommend different methods of fixation. Moreover, staining methods largely differ and imply Papanicolaou, hematoxylin eosin, or May-Grünwald-Giemsa [3,10,22-27].

During a continuous effort to improve the results of EUS-FNAC in our own clinic, we changed several parameters (e.g., needles of different sizes and shapes, number of needle passes and smears, puncture with and without vacuum, etc.) regarding the technical process of specimen acquisition. None of these measures, however, yielded substantial improvement. As a consequence, we discussed the issue with the local pathologist and agreed upon sending FNAC smears to an external specialized cytologist. Although we continue to obtain excellent histopathological results of core biopsies from the local pathologist, the evaluation of FNAC smears by a specialized cytologist had a striking effect on the diagnostic performance. In particular, sensitivity exceeded $90 \%$ and therefore equaled published data.
In most cases, we perform EUS-FNAC of solid pancreatic lesions to prove malignancy prior to initiating palliative chemotherapy or chemoradiotherapy. For this reason, the pretest probability of adenocarcinoma was quite high in the relatively large tumors (mean size $30 \mathrm{~mm}$ ) in this group of patients. Nevertheless, previous publications that addressed the statistical issues of EUSFNAC investigated tumors of similar sizes and are therefore comparable with our study [3-10].

Due to the fact that the technical aspects of EUS-FNAC are largely standardized (or have only a minor impact on sensitivity and specificity), preparation of smears and microscopic assessment remain the most variable factors for EUS-FNAC results. A retrospective study by Alsibai et al. has shown that an expert cytopathologist may considerably improve the sensitivity of EUS-FNAC as compared with local cytopathologists [27]. A similar effect has been demonstrated for the training of on-site cytotechnicians present during EUS: Diagnostic accuracy of smear evaluation increased from 74.8 to $90.5 \%$ after 1 year of training, involving more than 100 patients [25]. Recently, a German survey among more than 100 EUS centers demonstrated sobering results regarding the outcome of EUS-FNAC - more than half of the institutions reported a success rate of $75 \%$ and lower [28].

\section{Conclusions}

$\nabla$

Summing up, specialized cytologists should be involved if ambiguous or inadequate results are encountered despite optimization of specimen-acquisition technique. A sensitivity and specificity exceeding $90 \%$ can be achieved, but involves a well-balanced system of cell acqusition, preparation of smears, and further processing in the cytological laboratory. The Papanicolaou Society of $\mathrm{Cy}$ topathology has recently formulated guidelines for the terminology and nomenclature in pancreatobiliary cytology [29]. In the future, further consensual standards are mandatory for both endoscopists and cytologists to ensure a widespread, consistent quality of EUS-FNAC.

\section{Competing interests: None}


Institutions

${ }^{1}$ Department of Gastroenterology, Hepatology, and Gastroenterological Oncology, Bogenhausen Academic Teaching Hospital, Munich Municipal Hospital Holding, Munich, Germany

2 Department of Pathology, Bogenhausen Academic Teaching Hospital, Munich Municipal Hospital Holding, Munich, Germany

${ }^{3}$ Institute of Cytopathology, Hannover, Germany

\section{References}

1 Eltoum IA, Alston EA, Roberson J. Trends in pancreatic pathology practice before and after implementation of endoscopic ultrasound-guided fine-needle aspiration: an example of disruptive innovation effect? Arch Pathol Lab Med 2012; 136: 447-453

2 Dumonceau JM, Polkowski M, Larghi A et al. Indications, results, and clinical impact of endoscopic ultrasound (EUS)-guided sampling in gastroenterology: European Society of Gastrointestinal Endoscopy (ESGE) clinical guideline. Endoscopy 2011; 43: 897-912

3 Itoi T, Itokawa F, Sofuni A et al. Puncture of solid pancreatic tumors guided by endoscopic ultrasonography: a pilot study series comparing Trucut and 19-gauge and 22-gauge aspiration needles. Endoscopy 2005; 37: 362-366

4 Moller K, Papanikolaou IS, Toermer T et al. EUS-guided FNA of solid pancreatic masses: high yield of 2 passes with combined histologic-cytologic analysis. Gastrointest Endosc 2009; 70: 60-69

5 Takahashi K, Yamao K, Okubo K et al. Differential diagnosis of pancreatic cancer and focal pancreatitis by using EUS-guided FNA. Gastrointest Endosc 2005; 61: 76-79

6 Iglesias-Garcia J, Dominguez-Munoz E, Lozano-Leon A et al. Impact of endoscopic ultrasound-guided fine needle biopsy for diagnosis of pancreatic masses. World J Gastroenterol 2007; 13: 289-293

7 Varadarajulu S, Tamhane A, Eloubeidi MA. Yield of EUS-guided FNA of pancreatic masses in the presence or the absence of chronic pancreatitis. Gastrointest Endosc 2005; 62: 728 - 736; quiz 751, 753

8 Eloubeidi MA, Varadarajulu S, Desai $S$ et al. A prospective evaluation of an algorithm incorporating routine preoperative endoscopic ultrasound-guided fine needle aspiration in suspected pancreatic cancer. J Gastrointest Surg 2007; 11: 813-819

9 Hwang CY, Lee SS, Song TJ et al. Endoscopic ultrasound guided fine needle aspiration biopsy in diagnosis of pancreatic and peripancreatic lesions: a single center experience in Korea. Gut Liver 2009; 3: 116 - 121

10 Fisher L, Segarajasingam DS, Stewart $C$ et al. Endoscopic ultrasound guided fine needle aspiration of solid pancreatic lesions: performance and outcomes. J Gastroenterol Hepatol 2009; 24: 90-96

11 Fabbri C, Polifemo AM, Luigiano C et al. Endoscopic ultrasound-guided fine needle aspiration with 22- and 25-gauge needles in solid pancreatic masses: a prospective comparative study with randomisation of needle sequence. Dig Liver Dis 2011; 43: 647-652

12 Sakamoto H, Kitano M, Komaki $T$ et al. Prospective comparative study of the EUS guided 25-gauge FNA needle with the 19-gauge Trucut needle and 22-gauge FNA needle in patients with solid pancreatic masses. J Gastroenterol Hepatol 2009; 24: 384-390

13 Varadarajulu S, Bang JY, Hebert-Magee S. Assessment of the technical performance of the flexible 19-gauge EUS-FNA needle. Gastrointest Endosc 2012; 76: 336-343

14 Bang JY, Hebert-Magee S, Trevino J et al. Randomized trial comparing the 22-gauge aspiration and 22-gauge biopsy needles for EUS-guided sampling of solid pancreatic mass lesions. Gastrointest Endosc 2012; 76: $321-327$

15 Varadarajulu S, Fraig M, Schmulewitz $N$ et al. Comparison of EUS-guided 19-gauge Trucut needle biopsy with EUS-guided fine-needle aspiration. Endoscopy 2004; 36: 397-401

16 Hartwig W, Schneider L, Diener MK et al. Preoperative tissue diagnosis for tumours of the pancreas. Br J Surg 2009; 96: 5-20

17 Yoshinaga S, Suzuki H, Oda I et al. Role of endoscopic ultrasound-guided fine needle aspiration (EUS-FNA) for diagnosis of solid pancreatic masses. Dig Endosc 2011; 23: 29-33

18 Jenssen C, Dietrich CF. Endoscopic ultrasound-guided fine-needle aspiration biopsy and trucut biopsy in gastroenterology - an overview. Best Pract Res Clin Gastroenterol 2009; 23: 743 - 759

19 Eloubeidi MA, Varadarajulu S, Desai $S$ et al. Value of repeat endoscopic ultrasound-guided fine needle aspiration for suspected pancreatic cancer. J Gastroenterol Hepatol 2008; 23: 567-570

20 Tadic M, Kujundzic M, Stoos-Veic T et al. Role of repeated endoscopic ultrasound-guided fine needle aspiration in small solid pancreatic masses with previous indeterminate and negative cytological findings. Dig Dis 2008; 26: $377-382$

21 Cherian PT, Mohan P, Douiri A et al. Role of endoscopic ultrasoundguided fine-needle aspiration in the diagnosis of solid pancreatic and peripancreatic lesions: is onsite cytopathology necessary? HPB (Oxford) 2010; 12: 389-395

22 Uehara $H$, Ikezawa K, Kawada $N$ et al. Diagnostic accuracy of endoscopic ultrasound-guided fine needle aspiration for suspected pancreatic malignancy in relation to the size of lesions. J Gastroenterol Hepatol 2011; 26: 1256-1261

23 Lin F, Staerkel G. Cytologic criteria for well differentiated adenocarcinoma of the pancreas in fine-needle aspiration biopsy specimens. Cancer 2003; 99: 44-50

24 Mitsuhashi T, Ghafari S, Chang CY et al. Endoscopic ultrasound-guided fine needle aspiration of the pancreas: cytomorphological evaluation with emphasis on adequacy assessment, diagnostic criteria and contamination from the gastrointestinal tract. Cytopathology 2006; 17: $34-41$

25 Petrone MC, Arcidiacono PG, Carrara S et al. Does cytotechnician training influence the accuracy of EUS-guided fine-needle aspiration of pancreatic masses? Dig Liver Dis 2012; 44: 311-314

26 Eloubeidi MA, Chen VK, Eltoum IA et al. Endoscopic ultrasound-guided fine needle aspiration biopsy of patients with suspected pancreatic cancer: diagnostic accuracy and acute and 30-day complications. Am J Gastroenterol 2003; 98: 2663-2668

27 Alsibai KD, Denis B, Bottlaender J et al. Impact of cytopathologist expert on diagnosis and treatment of pancreatic lesions in current clinical practice. A series of 106 endoscopic ultrasound-guided fine needle aspirations. Cytopathology 2006; 17: 18-26

28 Barreiros AP, Burmester E, Will $U$ et al. Clinical procedures and outcomes of endoscopic fine needle biopsies (EUS-FNB): results of a German survey of the working group endoscopic ultrasound of the DEGUM. Ultraschall Med 2013: 34-WS_SL17_02

29 Pitman MB, Centerno BA, Ali SZ et al. Standardized terminology and nomenclature for pancreatobiliary cytology: the Papanicolaou Society of Cytopathology guidelines Diagn Cytopathol 19. 02. 2014: DOI $10.1002 / d c .23092$ 